\title{
Colliding impulsive waves in succession
}

\author{
M. Halilsoy \\ Physics Department, EMU, G. Magosa, Mersin 10, Turkey
}

(Received 14 May 1990)

\begin{abstract}
We formulate the initial-value problem for two colliding trains of impulsive gravitational waves. In the absence of a global, exact solution we show that in a region lying between the singularity $u^{2}+v^{2}=1$ and the wave trains, the solution is still the well-known Khan-Penrose solution.
\end{abstract}

\section{INTRODUCTION}

In a recent paper $^{1}$ we have introduced the formulation of the problem of colliding superposed waves in general relativity. Being unable to present an exact solution, we have constructed the proper initial data in the incoming regions. The formation of an essential and therefore impenetrable singularity, however, by the collision of the very first front waves raises the question of whether a physically acceptable solution exists at all. In this Brief Report we consider an arbitrary number of successive impulsive gravitational waves in collision and provide a new approach to this particular problem. The amplitude constants of the waves are chosen in such particular values that the original singularity obtained by Khan and Penrose $^{2,3}$ (KP) remains effective. Then, we observe that the original KP geometry is retained in a smaller region of space-time formed by successive waves located at $u<1 / \sqrt{2}(v<1 / \sqrt{2})$. The reason for this restriction is connected with the fact that for $u(v)>1 / \sqrt{2}$, the spacetime region to be obtained (as described below) falls beyond the essential singularity $u^{2}+v^{2}=1$, and therefore such incoming waves can be handled only within the context of an exact solution. A globally exact solution is beyond our scope and this handicap compels us to explore the possible validity of the KP solution for such a sequence of incoming waves.

\section{ARBITRARY NUMBER OF INCOMING WAVES}

In the harmonic coordinate system the space-time line element that describes gravitational plane waves ( $p p$ waves) is given by

$d s^{2}=2 d U d V-d X^{2}-d Y^{2}+h(U)\left(Y^{2}-X^{2}\right) d U^{2}$,

where the function $h(U)$ determines the profile of the waves. We shall choose now

$$
h(U)=\delta(U)+\sum_{i=0}^{n} A_{i} \delta\left(U-U_{i}\right),
$$

where $A_{i}$ are constant coefficients and $U_{i}$ denotes the location of the $i$ th impulsive wave. For $A_{i}=0$, we have $h(U)=\delta(U)$, which is the single wave case that gives rise to the KP solution. By this choice we assume an order- ing in the impulses such that $U_{i}>U_{j}$ whenever $i>j$. We apply now the coordinate transformation

$$
\begin{aligned}
& U=u, \quad X=x F, \quad Y=y G, \\
& V=v+\frac{1}{2}\left(x^{2} F F_{u}+y^{2} G G_{u}\right),
\end{aligned}
$$

which brings the line element into the Rosen form

$$
d s^{2}=2 d u d v-(F d x)^{2}-(G d y)^{2},
$$

and where the equations satisfied by $F$ and $G$ are

$$
\begin{aligned}
& F_{u u}=h(u) F, \\
& G_{u u}=-h(u) G .
\end{aligned}
$$

Solutions for $F$ and $G$ are given by

$$
\begin{aligned}
& F(u)=\mathcal{L}^{-1}\left(\frac{1}{s^{2}}+\frac{1}{s}+\sum_{i=0}^{n} A_{i} \frac{e^{-s u_{i}}}{s^{2}} F\left(u_{i}\right)\right), \\
& G(u)=\mathcal{L}^{-1}\left(-\frac{1}{s^{2}}+\frac{1}{s}-\sum_{i=0}^{n} A_{i} \frac{e^{-s u_{i}}}{s^{2}} G\left(u_{i}\right)\right),
\end{aligned}
$$

where $\mathcal{L}^{-1}\{\}$ denotes the inverse Laplace transform and $F\left(u_{i}\right), G\left(u_{i}\right)$ are the constant values for $F(u)$ and $G(u)$ at the locations of the $i$ th impulse. We have a similar metric for the other incoming region in which we substitute $u \rightarrow v, u_{i} \rightarrow v_{i}$, and $n \rightarrow m$. Although the number of waves can be arbitrarily chosen, for symmetry reasons, we shall make the choices $u_{i}=v_{i}$ and $m=n$, which imply that the two incoming regions involve the same number of waves located at equal intervals.

The constants $F\left(u_{l}\right)$ and $G\left(u_{i}\right)$ are given by the recurrence relations

$$
\begin{aligned}
& F\left(u_{n}\right)=1+u_{n}+\sum_{i=0}^{n-1} A_{i}\left(u_{n}-u_{i}\right) F\left(u_{i}\right), \\
& G\left(u_{n}\right)=1-u_{n}+\sum_{i=0}^{n-1} A_{i}\left(u_{n}-u_{i}\right) G\left(u_{i}\right),
\end{aligned}
$$

where for $n=0$ we have $F\left(u_{0}\right)=1+u_{0}$ and $G\left(u_{0}\right)=1-u_{0}$. In the case of a single impulsive wave at $u=0$, we have $u_{0}=0$, which leads to the conditions $F(0)=1=G(0)$. We rewrite the incoming space-time line element (4) in the form 


$$
d s^{2}=2 d u d v-e^{-U}\left(e^{V} d x^{2}+e^{-V} d y^{2}\right),
$$

where $e^{-U}=F G$ and $e^{V}=F / G$. We want now to choose $e^{-U}$ the same as in the single wave case, namely,

$$
e^{-U}=1-u^{2} \theta(u)
$$

and fix all the constants accordingly. In order to achieve this, we multiply $F(u)$ by $G(u)$ and equate the result to $1-u^{2} \theta(u)$, which determines all $A_{i}$ :

$$
A_{i}=\frac{2(-1)^{i+1}}{1-u_{i}^{2}} \quad(i=0,1,2, \ldots, n-1) .
$$

Using these constants and the values for $F\left(u_{n}\right), G\left(u_{n}\right)$ from (7) we express the incoming region metrics in terms of an arbitrary number of waves. For the three-wave case, as an example, we have the incoming metric functions

$$
e^{-U}=1-u^{2} \theta(u), \quad e^{V}=\frac{F}{G},
$$

where

$$
\begin{aligned}
F= & 1+u \theta(u)-\frac{2}{1-u_{0}}\left(u-u_{0}\right) \theta\left(u-u_{0}\right) \\
& +\frac{2}{1-u_{0}} \frac{1+u_{0}}{1+u_{1}}\left(u-u_{1}\right) \theta\left(u-u_{1}\right), \\
G= & 1-u \theta(u)+\frac{2}{1+u_{0}}\left(u-u_{0}\right) \theta\left(u-u_{0}\right) \\
& -\frac{2}{1+u_{0}} \frac{1-u_{0}}{1-u_{1}}\left(u-u_{1}\right) \theta\left(u-u_{1}\right) .
\end{aligned}
$$

The three impulsive waves, obviously, are located at the points $\left(0, u_{0}, u_{1}\right)$, respectively, and the expressions are suggestive for a straightforward generalization. The $F, G$ functions for an arbitrary number of waves are found without much effort as

$$
\begin{aligned}
F(u)=1+u \theta(u)-2 \sum_{l=0}^{n} & (-1)^{l}\left(u-u_{l}\right) \theta\left(u-u_{l}\right) \\
& \times\left[\prod_{k=0}^{l}\left[\frac{1+(-1)^{k-1} u_{k-1}}{1-(-1)^{k} u_{k}}\right]\right],
\end{aligned}
$$$$
G(u)=1-u \theta(u)+2 \sum_{l=0}^{n}(-1)^{l}\left(u-u_{l}\right) \theta\left(u-u_{l}\right)
$$$$
\times\left[\prod_{k=0}^{l}\left[\frac{1+(-1)^{k} u_{k}-1}{1-(-1)^{k+1} u_{k}}\right]\right],
$$

where $u_{-1}=0$. It can be checked that these functions satisfy $F G=1-u^{2} \theta(u)$, and a similar construction for the other incoming region provides $e^{-U}=1-v^{2} \theta(v)$. Being combined together, the two incoming regions suggest that in the interaction region we can choose

$$
e^{-U}=1-u^{2}-v^{2}=e^{-U_{\mathrm{KP}}},
$$

which is essentially the choice that we abide by.

\section{THE PHYSICAL INTERPRETATION}

As admitted in the beginning it is not our intention here to seek for an interaction region metric that will match the $F, G$ components in (12). Rather, by special choices of the coefficients we have rendered the condition (13) for one of the metric functions. For a symmetrical collision problem we impose now the condition that all successive waves are located in a region $0 \leq u<1 / \sqrt{2}$ and $0 \leq v<1 / \sqrt{2}$. Otherwise, for incoming waves satisfying $u(v)>1 / \sqrt{2}$, the extrapolation of the incoming waves into the interaction region leads to a meeting point which is beyond the singularity $u^{2}+v^{2}=1$, and therefore we discard it from our discussion. Once we impose this condition on our initial data, which is always possible, it enables us to determine the function $e^{V}=F / G$ in the interaction region. To this end, we express $V(u)$ in the incoming region after some manipulation as

$$
\begin{aligned}
& e^{V}=\prod_{k=1}^{2 n-1}\left(\frac{1-(-1)^{k} u_{k-1}}{1+(-1)^{k} u_{k-1}}\right) \times \begin{cases}\frac{1-u}{1+u} & \text { for } 2 n \text { waves } \\
\frac{1+u}{1-u} & \text { for } 2 n+1 \text { waves }\end{cases} \\
& =\alpha\left(\frac{1 \mp u}{1 \pm u}\right),
\end{aligned}
$$

where the constant factor of products is denoted in short by $\alpha$. A similar expression exists with $u \rightarrow v$ and $u_{i} \rightarrow v_{i}=u_{i}$ for the other incoming region. This form of $e^{V}$ can be recognized now (with $\alpha=1$ ) as the KP form. The interaction region line element is

$$
d s^{2}=2 e^{-M} d u d v-e^{-U}\left(e^{V} d x^{2}+e^{-V} d y^{2}\right),
$$

where

$$
\begin{aligned}
& U=U_{\mathrm{KP}}, \quad M=M_{\mathrm{KP}}, \\
& e^{V}=\alpha e^{ \pm V_{\mathrm{KP}}}=\alpha\left[\frac{1 \pm \tau}{1 \mp \tau}\right), \\
& \tau=u\left(1-v^{2}\right)^{1 / 2}+v\left(1-u^{2}\right)^{1 / 2}, \\
& \alpha=\text { const } \quad[\text { in Eq. (14)], }
\end{aligned}
$$

in which $+(-)$ refer to the even (odd) number of waves and KP refers to the Khan-Penrose functions. The validity domain of this solution, however, is

$$
\begin{aligned}
& u>u_{2 n-1}, v>v_{2 n-1}, u^{2}+v^{2} \leq 1 \text { for } 2 n \text { waves, } \\
& u>u_{2 n}, v>v_{2 n}, u^{2}+v^{2} \leq 1 \text { for } 2 n+1 \text { waves. }
\end{aligned}
$$

In conclusion, this interpretation of colliding waves in succession does not contradict any physical condition and, unlike the problem of colliding superposed electromagnetic waves, ${ }^{4}$ crossing of the singularity does not take place. In the latter case, we recall that an exact solution exists globally but waves have to cross into nonallowable regions consisting of $\delta$ function curvatures at the boundaries. ${ }^{4}$ In a linearized theory only, where the $\delta$ functions are approximated by appropriate peaks, the solution of colliding electromagnetic waves may have some physical use.
${ }^{1}$ M. Halilsoy, Phys. Rev. D 39, 2172 (1989).

${ }^{2}$ K. A. Khan and R. Penrose, Nature (London) 229, 185 (1971).

${ }^{3}$ P. Szekeres, J. Math. Phys. 13, 286 (1972).
${ }^{4}$ M. Gurses and M. Halilsoy, Lett. Nuovo Cimento 34, 588 (1982). 